\title{
The effect of patient technique and training on the accuracy of self-recorded peak expiratory flow
}

\author{
P.F.G. Gannon*, J. Belcher ${ }^{+}$, C.F.A. Pantin ${ }^{+}$, P.S. Burge*
}

\begin{abstract}
The effect of patient technique and training on the accuracy of self-recorded peak expiratory flow. P.F.G. Gannon, J. Belcher, C.F.A. Pantin, P.S. Burge. ERS Journals Ltd 1999.

ABSTRACT: The aim of the study was to investigate the difference between encouraged self-recorded peak expiratory flow (PEF) with unobserved readings and to investigate any long-term changes in PEF self-recording.

Patients were trained in the PEF technique and asked to keep 2-hourly PEF records until the next clinic visit. The patients PEF were then rechecked at the second clinic visit by a series of two unobserved, an observed and an encouraged PEF measurement. A subgroup of patients were reassessed at a third clinic visit.

Forty-one patients produced serial PEF readings. Significant differences between unobserved and encouraged PEF readings were detected; there was a mean decrement of $21 \mathrm{~L} \cdot \mathrm{min}^{-1}$ and limits of agreements suggested that the decrement could be as high as $60 \mathrm{~L} \cdot \mathrm{min}^{-1}$. Visual and statistical analysis of the serial PEF provided showed a consistent deterioration in PEF over the record in 54\% and $39 \%$ of cases, respectively. No significant differences were found in the subgroup who attended a third clinic visit.

The results suggest that significant inaccuracies in unobserved peak expiratory flow readings can occur between clinic visits and this can be reflected as a consistent deterioration in some. This should be kept in mind when interpreting self-recorded peak expiratory flow measurements. Re-evaluation at the third visit following the retraining effect of the second visit on peak expiratory flow technique appears to reduce inaccuracies. It is believed that peak expiratory flow technique should be reevaluated at each clinic visit.
\end{abstract}

Eur Respir J 1999; 14: 28-31.

\begin{abstract}
*Occupational Lung Disease Unit, Birmingham Heartlands Hospital, Birmingham, UK. 'Industrial and Community Health Research Centre, Keele University School of Post Graduate Medicine, North Staffordshire Hospital, Stoke-onTrent, UK
\end{abstract}

Correspondence: P.S. Burge

Occupational Lung Disease Unit Birmingham Heartlands Hospital Bordesley Green

East Birmingham B9 5SS

UK

Fax: 441217720292

Keywords: Asthma

occupational asthma

peak expiratory flow

quality control

Received: November 51997

Accepted after revision January 191999
Serial self-recorded peak expiratory flow (PEF) readings are widely used in the diagnosis, investigation and management of asthma and chronic airflow limitation. A number of problems have been described with measurement of PEF. MAY and JANIC [1] studied PEF measurements performed by Polish military recruits, grading for musculature and degree of co-operation. They found significantly higher PEF readings in those deemed to have greater muscle mass versus those without. They also found significantly higher PEF readings in those with good or very good co-operation versus those whose cooperation was graded as only satisfactory or bad. ALLEN et al. [2] reported the positive effort dependence of maximal expiratory flow, concluding that flow can increase with effort over a large proportion of vital capacity. Other authors $[3,4]$ have described ways of cheating the peak flow meter to produce artificially high peak flow readings by acceleration in the mouth.

Hetzel et al. [5] found that in a group of asthmatic patients $69 \%$ of unobserved PEF readings in hospital were within $10 \%$ of observed readings and concluded that self-monitored PEF readings were practical and of sufficient accuracy for clinical use.

TROYANOv et al. [6] looked at the accuracy of PEF readings in terms of meeting recognized reproducibility standards for PEF readings in a study of 29 subjects (19 asthmatics) in which it was found that $78 \%$ of the PEF values recorded were reproducible to American Thoracic Society criteria [7] (the best of two reproducible values $\left.\pm 20 \mathrm{~L} \cdot \mathrm{min}^{-1}\right)$.

A further consideration when analysing self-recorded PEF is demonstrated by other studies performed out of hospital concentrating on accuracy in terms of falsification of PEF readings and inaccuracies in the recorded timing of PEF readings. CHOWIENCZYK et al. [8], in a study of 33 adult asthmatics, used an electronic PEF meter capable of storing date and timed PEF readings, as well as a diary card. Using the electronic PEF meter, $86 \%$ of expected readings were produced compared to $70 \%$ using a diary card. Of the data entered onto the diary card, $4 \%$ of recorded entries had no counterpart on the logging meter and $22 \%$ were mistimed.

QuiRCE et al. [9], in a study of 17 subjects under investigation for occupational asthma, investigated their ability to perform unsupervised serial PEF measurements, again using a data logging electronic PEF meter. Twentythree per cent of patients were not able to complete a 4-week record; of the remainder, $55.3 \%$ of patient's readings were accurate in terms of date and time, $23.3 \%$ were inaccurate in terms of the timing of the reading or the recorded value. SiRACUSA et al. [10], in a study of response to treatment in 12 male workers with occupational asthma, 
found that $95.4 \%$ of expected PEF readings were produced over a 4-week recording period.

The aims of this study were to investigate the difference between self-recorded PEF under encouragement with measurements made without encouragement, and to investigate any long-term changes in PEF self-recording likely to be due to lack of effort.

\section{Materials and methods}

Consecutive patients were recruited from a general chest clinic, inclusion criteria were: patients of working age, with either asthma, chronic airflow limitation or nonobstructive chest pathology. Exclusion criteria were: patients who were either illiterate or had uncorrected impaired visual acuity or mental/physical problems that were likely to interfere with the study. Previous experience with the PEF meter was allowed. Signed consent to participate in the study was obtained.

At the first visit, the purpose of the study was explained, the patients were educated in the use of the Mini-Wright PEF meter (Airmed, Clement Clarke International Ltd., Essex, UK) using a written text (reproducibility criteria: best of three readings with the two highest within 20 $\mathrm{L} \cdot \mathrm{min}^{-1}$, recording only the highest reading) and their PEF technique was checked by a doctor. Technique was then rechecked by a pulmonary function technician, with further training if necessary. Patients were excluded if they were unable to achieve a satisfactory technique. Following successful training, patients were asked to perform measurements of PEF every $2 \mathrm{~h}$ during waking hours for 2-3 weeks and to record the readings on a standard form. No data was sought on whether the patients had taken bronchodilators before this visit.

On arrival at the second visit and after each set of PEF measurements, the patient rested for $10 \mathrm{~min}$; they were asked not to take $\beta_{2}$-agonists for $4 \mathrm{~h}$ prior to attending the clinic. The patient performed a set of PEF measurements unobserved in an outpatient side room (first unobserved $\mathrm{PEF}$ reading). PEF measurements were then performed, observed but not encouraged by a doctor (observed PEF reading). PEF measurements were then performed and actively encouraged by a pulmonary function technician (encouraged PEF reading). Finally, a second set of PEF measurements were performed unobserved (second unobserved PEF reading). Patients themselves read and wrote down all sets of PEF readings and the same PEF meter was used for all four sets of PEF measurements throughout the study by the individual patients. All unobserved and observed PEF readings were assessed for agreement with the encouraged PEF reading using the technique described by Bland and Altman [11]. This method can be used to assess agreement between two methods of clinical measurement. It calculates limits of agreement based on the mean difference and the standard deviation of the differences. The limits of agreement can be used to predict whether clinically significant differences in PEF are likely to occur. A Student's t-test was performed to determine the significance of the differences.

Three of the authors individually examined the PEF records produced by the patients between the first and the second visit. The records were plotted as maximum, mean and minimum for each day (fig. 1) and examined for signs of change in PEF likely to be due to lack of effort, such as an initial fall in PEF over the first few days of the record reaching a plateau for the remainder or a gradual deterioration in PEF over the whole record. Records were denoted as having either signs of a pattern of deterioration in PEF over their length or no pattern of deterioration, where PEF varied randomly throughout the record. When authors did not agree, a majority opinion prevailed.

The serial PEF readings were also analysed statistically by fitting a least squares linear trend line through the PEF data. Analysis of variance was used to determine the statistical significance of any trend detected.

A subgroup of patients participating in the study whose next routine clinic appointment occurred within the next year were seen when they next returned to clinic and were asked to repeat a third series of PEF measurements as performed at the second visit. These readings were again compared to the encouraged PEF value as described above.

\section{Results}

Forty-four patients agreed to participate in the study. Twenty-eight suffered with asthma, 10 with chronic airflow limitation and six with nonobstructive chest pathology. The mean age was 49 yrs (range 18-65 yrs); 26 $(53 \%)$ were males. The mean number of PEF readings per day was 7.8, and the number of days with at least four PEF readings was $95.7 \%$. There was very little difference between the first visit PEF readings performed for training with the doctor and those performed for checking with the technician, with a mean difference of $-2.3 \mathrm{~L} \cdot \mathrm{min}^{-1}$ and

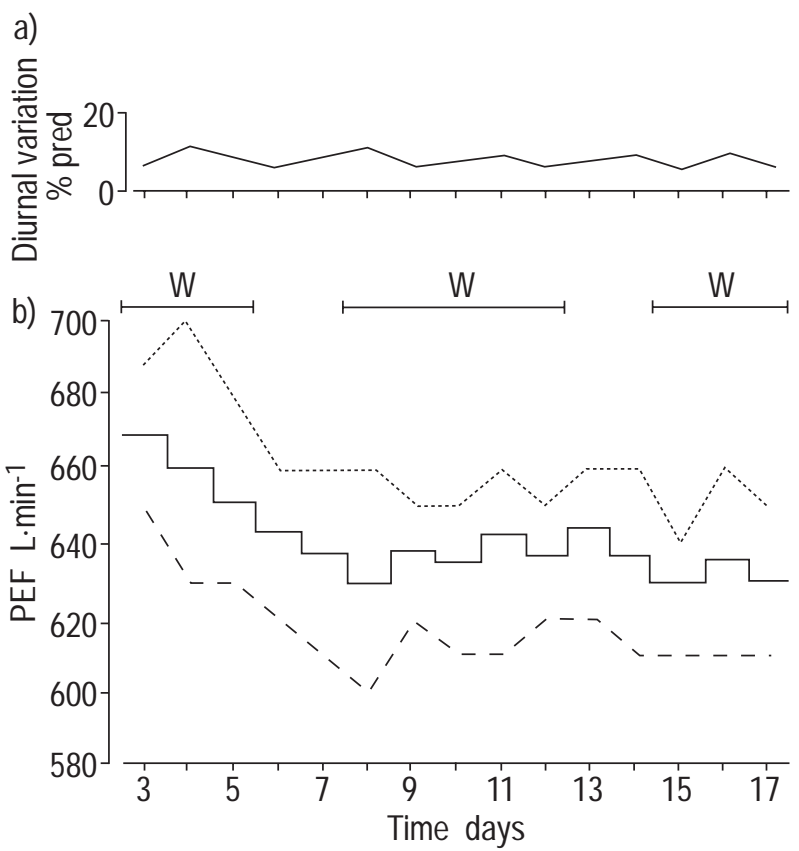

Fig. 1. - a) Diurnal variation (\% predicted) and b) peak expiratory flow (PEF) record plotted as maximum (- - - ), minimum (- - ) and mean (-) against time in days. The PEF record shows a deterioration in mean PEF over the first 5 days of the record. This patient's PEF readings on returning to the clinic were: first unobserved $670 \mathrm{~L} \cdot \mathrm{min}^{-1}$, observed $650 \mathrm{~L} \cdot \mathrm{min}^{-1}$, encouraged $690 \mathrm{~L} \cdot \mathrm{min}^{-1}$, second unobserved $690 \mathrm{~L} \cdot \mathrm{min}^{-1}$. The improvement of $20 \mathrm{~L} \cdot \mathrm{min}^{-1}$ with encouragement was consistent with the fall seen over the first 5 days of the unsupervised PEF record. $\mathrm{W}$ : days at work. 
Table 1. - Peak expiratory flow (PEF) readings of patients at second and third visits

\begin{tabular}{lccr}
\hline & Visit 2 (all) & Visit 2 (subgroup) & \multicolumn{1}{c}{ Visit 3} \\
\hline Mean PEF first unobserved L·min ${ }^{-1}$ & 411 & 380 & 373 \\
First unobserved versus encouraged & $-21(-60-19)^{*}$ & $-24(-67-18)^{*}$ & $-10(-57-37)$ \\
Observed versus encouraged & $-9(-46-27)^{*}$ & $-17(-55-21)^{*}$ & $4(-29-36)$ \\
Second unobserved versus encouraged & $-6(-36-23)$ & $-11(-43-21)^{*}$ & $-5(-44-34)$ \\
First versus second unobserved & $-15(-47-23)^{*}$ & $-14(-46-18)^{*}$ & $-5(-53-42)$ \\
\hline
\end{tabular}

Data presented as mean decrement and with limits of agreement in parentheses for comparison of unobserved, observed and encouraged $\mathrm{PEF}$ at the second and third clinic visits. The encouraged and second unobserved PEF reading acting as the standard measure against which the decrement is measured. A Student's t-test was used to determine the significance of the differences. *: $\mathrm{p}<0.05$.

limits of agreement between -32 and $28 \mathrm{~L} \cdot \mathrm{min}^{-1}$ using the PEF readings obtained with the technician as the true PEF. All patients had a satisfactory PEF technique on leaving the clinic after the first visit.

All patients returned at the second visit, with a mean time interval between the first and second visit of 17.5 days (range 7-28); 41 patients produced PEF records documenting PEF readings performed between the two visits. The results of the comparison between the second visit first unobserved, observed and second unobserved PEF readings and the encouraged PEF reading are shown in table 1. There was a significant difference between both the mean first unobserved PEF reading and the observed PEF reading compared to the encouraged PEF reading. There was a mean decrement of $21 \mathrm{~L} \cdot \mathrm{min}^{-1}$ for the first unobserved PEF reading and the limits of agreement suggested that the difference may be $60 \mathrm{~L} \cdot \mathrm{min}^{-1}$ below or 19 $\mathrm{L} \cdot \mathrm{min}^{-1}$ above the encouraged PEF reading. After retraining the second unobserved PEF reading was not significantly different from the encouraged PEF reading, but was significantly different from the first unobserved value. Comparison of the mean difference between first unobserved and encouraged for asthmatics (mean -24.6 $\mathrm{L} \cdot \mathrm{min}^{-1}, 95 \%$ confidence intervals (CI) $\left.-16.4--32.9\right)$ and nonasthmatics (mean -14.4 L. $\mathrm{min}^{-1}, 95 \% \mathrm{CI}-7.5-21.2$ ) showed no significant difference.

Forty-one patients returned PEF records carried out between the first and second visits; the results of visual and statistical analysis are shown in table 2 . On visual examination, $21(51 \%)$ cases showed a pattern of deterioration in PEF over the length of the record, an example of which is shown in figure 1. Statistical analysis showed significant linear trends over the record in $16(39 \%)$ cases. The mean difference between first unobserved and encouraged PEF readings for the group of 21 patients who showed a consistent deterioration by visual analysis (mean -17.4 L- $\min ^{-1}, 95 \%$ CI -9.9- -25.2) did not significantly differ from those who did not (mean $-25.5 \mathrm{~L} \cdot \mathrm{min}^{-1}$, $95 \%$ CI $-16.3--34.8)$.

Visual analysis detected three (7\%) PEF records which showed evidence suggestive of an acute exacerbation of airways obstruction during the record (duration 5-10 days). This was represented as a rapid fall in PEF over 2-3 days with a subsequent recovery over a period of days to the pre-existing PEF values; in all cases the exacerbation had recovered by the second clinic visit.

There was no significant difference in mean PEF difference when comparing the first unobserved with the encouraged reading at visit 2 between the 35 patients who had used the PEF meter before visit 1 (mean $-23.4 \mathrm{~L} \cdot \mathrm{min}^{-1}$, $95 \%$ CI $-16.5--30.4$ ) and the nine who had not (mean $-11.1 \mathrm{~L} \cdot \mathrm{min}^{-1}, 95 \%$ CI $\left.-2.2--20.0\right)$.

Nineteen patients returned for a third clinic visit and repeated the second visit procedure; the mean duration between visits was 4.7 months (range $2-13$ months). The mean age of the subgroup was 50 yrs (range 21-65), 63\% were male, 16 suffered from asthma and three from chronic airflow limitation. The results of the subgroup for the second and third visits are shown in table 1 .

For the second visit, significant differences were seen between the encouraged value and all the other PEF readings, and the mean decrement was increased compared to the study group as a whole. Comparison of the first and second unobserved PEF readings for the subgroup also showed a significant decrement; however, this was reduced compared to the decrement found for the group as a whole.

For the third visit there were no significant differences in PEF readings between the unobserved and observed PEF readings compared to the encouraged PEF reading, although the mean decrement was $10 \mathrm{~L} \cdot \mathrm{min}^{-1}$ for the first unobserved PEF reading and the limits of agreement suggested that it may be $57 \mathrm{~L} \cdot \mathrm{min}^{-1}$ below or $37 \mathrm{~L} \cdot \mathrm{min}^{-1}$ above the encouraged value. There was no significant difference between the first and second unobserved PEF readings.

\section{Discussion}

The 44 patients in this study were representative of attendees to a general adult chest clinic in terms of age, sex, and diagnosis and the results should therefore be applicable to other chest clinic patients. However, no normal control subjects were included, and thus strictly speaking the results apply to monitoring and not necessarily diagnosis of patients with chest pathology. The results suggest that the $93 \%$ of patients who returned PEF records were able to keep what appeared to be high quality records,

Table 2. - Results of visual and statistical analysis of serial peak expiratory flow (PEF) records for the presence of consistent deteriorations in PEF over the record

\begin{tabular}{|c|c|}
\hline & PEF records $n(\%)$ \\
\hline No consistent deterioration by visual or statistical analysis & $16(39)$ \\
\hline Consistent deterioration detected by visual and statistical analysis & $12(29)$ \\
\hline Consistent deterioration detected only by visual analysis & $9(22)$ \\
\hline Consistent deterioration detected only by statistical analysis & $4(10)$ \\
\hline
\end{tabular}

Agreement between all three visual interpreters was $83 \%$, overall agreement between visual and statistical analysis was $68 \%$. 
indicating that they had understood instructions on timing and recording of PEF readings. The finding that only $7 \%$ of patients were unable to keep serial PEF records was more favourable than the finding of $23 \%$ in the study by QUiRCE et al. [9]. Comment cannot be made on how accurate these PEF readings were in terms of falsification and accurate timing. However, an evaluation of their actual accuracy in terms of measurement of PEF can be inferred from the patients performance when attending the second clinic visit. The results from which suggest that after one set of training self-recorded PEF outside the hospital may be significantly different from PEF values produced by encouragement, the mean decrement being $21 \mathrm{~L} \cdot \mathrm{min}^{-1}$. Limits of agreement suggested that these differences may be clinically significant.

During the second and third visits control for true variation in PEF was not achieved which may have occurred because of exercise-induced changes in airways obstruction and fatigue during the 12 PEF efforts involved in the visits. The usual way of overcoming this problem, random order measurement, clearly could not be applied here. However, comparison of the first and second unobserved readings suggest that induced airways obstruction and fatigue was not a major problem, although the differences may have been shown to be greater had this not been a factor. This effect probably explained the minority of cases where the first unobserved reading was higher than the encouraged or second unobserved reading represented by the positive values in the limits of agreement shown in table 1 .

The consistent deterioration seen in between 39 and 54\% of the serial PEF measurements may be due to a number of factors other than worsening airways obstruction, including lack of effort, which may occur as soon as the patient leaves the clinic or may occur gradually over time away from the clinic, or for technical reasons such as the PEF meter sticking. The lack of effort hypothesis is supported by the fact that the three PEF records which were suggestive of acute exacerbation showed only short lived deterioration in $\mathrm{PEF}$, followed by recovery. This compared to the consistent deterioration shown in 54\% of PEF records (probably due to lack of effort), which was characterized by an immediate fall in PEF over the first few days reaching a plateau as shown in figure 1 , with no recovery.

It could be suggested that some of the deterioration seen between the first two visits is due to the request for 2hourly PEF readings, as is used in the investigation of occupational asthma, rather than 2-4 readings, as is requested for the normal management of asthma. However, the authors do not feel that this is the case as they have also recorded significant learning effects [12], with an increase in PEF over the record, in patients under the investigation of occupational asthma and performing 2-hourly PEF readings.

The inconsistent deterioration seen in between 46 and $61 \%$ of the serial PEF measurements may be due to a patient whose effort is inconsistent; this may be interpreted as a falsely high diurnal variation suggesting uncontrolled asthma. These factors need to be considered when interpreting these PEF records and reference made to the patient's PEF technique when they return before conclusions are drawn from a PEF record. In terms of an effect on the diagnosis of occupational asthma, an important confounding effect should only be seen if effort varies consistently between days at and away from work. This may occur if a patient deliberately tries to fabricate a PEF record to suggest occupational asthma.

After further retraining in PEF technique, during the encouraged PEF measurement at visit 2, significant differences did not occur between unobserved and encouraged readings at the third visit suggesting that good technique had been preserved after the second visit. This was despite a generally poorer performance of the subgroup at the second visit compared to the group as a whole.

The fact that some of the patients in the study already used PEF meters prior to the first visit, and whose technique deteriorated prior to their second visit suggests that the improvement seen at the third visit may not be longterm.

These results suggest that patients should have their peak expiratory flow technique checked at each clinic visit with retraining as necessary if the technique has deteriorated. Patients who demonstrate poor technique should have their serial peak expiratory flow records considered carefully for effects which may be due to technique rather than true airway changes.

\section{References}

1. May K, Janic G. Effect of patient co-operation and muscular physique on maximum expiratory flow rate. Pneumonologia Polska 1985; 53: 53-56.

2. Allen JL, Castile RG, Mead J. Positive effort dependence of maximum expiratory flow. $J$ Appl Physiol 1987; 62: 718-724.

3. Connolly CK. Falsely high peak expiratory flow readings due to acceleration in the mouth. BMJ 1987; 294: 285.

4. Colin A, Said E. How to cheat the Mini-Wright peak flow meter (letter). Chest 1984; 86: 156.

5. Hetzel MR, Williams IP, Shakespeare RM. Can patients keep their own peak flow reliably? Lancet 1979; i: $597-$ 599.

6. Troyanov S, Ghezzo H, Cartier A, Malo JL. Comparison of circadian variations using FEV1 and peak expiratory flow rates among normal and asthmatic subjects. Thorax 1994; 49: 775-780.

7. American Thoracic Society. Standardization of spirometry-1987 update. Am Rev Respir Dis 1987; 136: 12851307.

8. Chowienczyk PJ, Parkin DH, Lawson CP, Cochrane GM. Do asthmatic patients correctly record home spirometry measurements. BMJ 1994; 309: 1618.

9. Quirce S, Contreas G, Dybuncio A, Chan-Yeung M. Peak expiratory flow monitoring is not a reliable method for establishing the diagnosis of occupational asthma. $\mathrm{Am} \mathrm{J}$ Respir Crit Care Med 1995; 152: 1100-1102.

10. Siracusa A, Forcina A, Brugnami G, Ranciaro A, Fiordi $\mathrm{T}$, Marabini A. Monitoring of peak expiratory flow rates during occupational asthma treatment. Medicina del Lavoro 1992; 83: 157-166.

11. Bland JM, Altman DG. Statistical method for assessing agreement between 2 methods of clinical measurement. Lancet 1986; i: 307-310.

12. Gannon PFG, Burge PS. Serial peak expiratory flow in the diagnosis of occupational asthma. Eur Respir J 1997; 10: Suppl. 24, 57s-63s. 